\title{
Fuzzy-PID Controller Optimization: A Differential Evolution Approach
}

\section{Ana Sebastião ${ }^{1}$, Luís Palma ${ }^{1,2}$, Alberto Cardoso³, Paulo Gil ${ }^{1,2,3}$}

${ }^{1}$ Department o de Engenharia Electrotéctica, Faculdade de Ciências e Tecnologia, Universidade Nova de Lisboa, Portugal

${ }^{2}$ CTS-UNINOVA, Campus of FCT/UNL, Portugal

${ }^{3}$ CISUC, Dep. of Informatics Engineering, FCTUC, Portugal

am.sebastiao@campus.fct.unl.pt, lbp@fct.unl.pt, alberto@dei.uc.pt,psg@fct.unl.pt

Keywords: Fuzzy PID Control, Tuning, Constrained Nonlinear Optimisation, Differential Evolution.

\begin{abstract}
This paper addresses the problem of Fuzzy Proportional-Integral-Derivative control tuning based on a differential evolution technique. Two different constrained optimisation approaches are considered. One where solely the controller scaling factors are adjusted, and another for which both the scaling factors and membership functions width are optimised. The cost function is expressed in terms of the system closed loop performance, with the plant dynamics described by a nonlinear model. The formulation includes a set of constraints, namely bounds on scaling factors, control actions and on the system outputs. For the second approach additional constraints for the membership functions width are also considered. Experimental results carried out on a Multi-Input Multi-Output benchmark system favour the optimisation of both gains and membership functions width.
\end{abstract}

\section{Introduction}

Proportional-Integral-Derivative (PID) control is still in these days widely used in industry. The main motivation for using this type of controllers is to some extend due to its functional/structural simplicity, easy tuning and low cost implementation. When the system dynamics exhibits higher nonlinearities or in the case of time varying dependencies, such technique invariably leads to under performance or even instability. A very intuitive nonlinear technique takes advantage of fuzzy logic theory for designing control structures [1], in particular those represented by Mamdani type architectures.

In the present study the differential evolution (DE) method is considered for tuning both the scaling factors and member ship functions width. Although other versions based on the scaling factors optimisation have been proposed and evaluated (see e.g. [2]), the incorporation of adaptation mechanisms regarding the membership functions width is somehow a novelty to the best of the authors knowledge. Hence, this work aims at comparing the outcomes in terms of closed loop performance in two different architectures. The first considers the scaling factors tuning, with the membership functions width unchanged, while in the second approach both the scaling factors and the membership functions width are also optimised. In both approaches, the optimisation is carried out offline, and includes constraints on the controller scaling factors, control actions, increment of control actions and outputs. The plant dynamics is approximated by a Nonlinear Autoregressive with Exogenous Inputs (NARX) neuronal network. Additionally, in the second scheme to optimisation problem also includes a set of constraints for the membership functions.

\section{Fuzzy Logic Control Systems}

The basic structure of a FLC system consists of four conceptual components: the knowledge base, fuzzification interface, inference engine, and finally the defuzzification interface. The knowledge base module contains all the required controller's knowledge/information, namely the rule base and a data base. The inference engine is a reasoning mechanism that performs inference operations upon the fuzzy control rules, and given operating conditions, in order to provide control 
actions as outputs. The fuzzification component defines a mapping from a real-valued (crisp) subspace to a fuzzy one, while the defuzzification module implements a mapping from a fuzzy subspace, defined over an output universe of discourse to a real-valued subspace (crisp) [1].

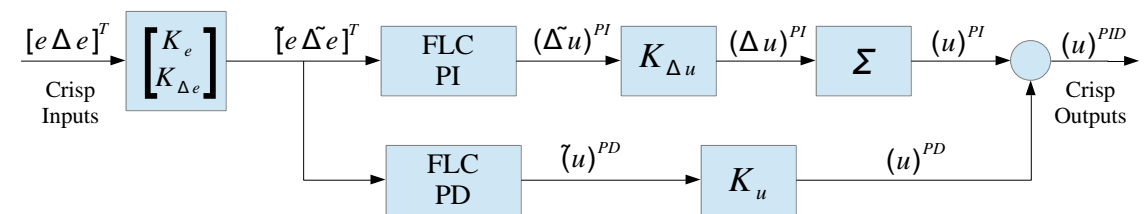

Fig.1: Fuzzy-PID controller schematics.

PID-Fuzzy Controller Architecture. The fuzzy-PID control topology considered in this work consist of two-input single-output PID-fuzzy controller structure, as shown in Fig. 1, where $K_{e}$, $K_{\Delta e}, K_{\Delta \mathrm{u}}$ and $K_{\mathrm{u}}$ are the controller scaling factors. This architecture assumes as input variables the error $e$ (1) and the change in error $\Delta e$ (2), and delivers as output the control action $u^{P I D}$,

$$
\begin{aligned}
& e(k)=r(k)-y(k) \\
& \Delta e(k)=e(k)-e(k-1)
\end{aligned}
$$

with $k$ the current discrete time, $y$ the system output and $r$ the reference signal. The normalised error $\tilde{e}$, change in error $\Delta \tilde{e}$, as well as the denormalised control action $u^{P D}$ and the increment of control action $\Delta u$ are given by:

$$
\begin{aligned}
& \tilde{e}(k)=k_{e} \cdot e(k) \\
& \Delta \tilde{e}(k)=k_{\Delta e} \cdot \Delta e(k) \\
& \Delta u^{P I}(k)=k_{\Delta u} \cdot \Delta \tilde{u}^{P I} \\
& u^{P D}(k)=k_{u} \cdot \tilde{u}^{P D}
\end{aligned}
$$

where $\Delta \tilde{u}^{P I}(k)$ is the normalised increment of control action associated with the fuzzy PI block and $\tilde{u}^{P D}(k)$ is the normalised fuzzy PD contribution.

Finally, it should be mentioned that different fuzzy Mamdani-type controller architectures can be considered, having no impact on the proposed general tuning methodology.

Differential Evolution Based Tuning. The DE is a powerful evolutionary algorithm for global optimization in real problems. This technique is a parallel direct search method, which uses NP $D$ dimensional parameter vectors $x_{i, G}, i=1,2, \ldots, N P$ as a population, for each generation $G$ [3], with $N P$ the number of parents, which are kept unchanged over the minimisation process. The initial vector population is chosen randomly and should cover the whole parameter space. In the optimisation process the DE generates new parameter vectors by adding the weighted difference between two population vectors to a third vector. If the trial vector has a lower cost function value than that of the target vector, the trial vector will replace the target vector in the next generation, being this operation denoted as selection. Additionally, each population vector has to serve once as the target vector so that $N P$ competitions take place in one generation. Fig. 2 shows the modus operandi of the DE technique. The reader is referred to [4] and references there in for a thoroughly introduction to this method.

Gains Optimization. In both approaches the scaling factors of the fuzzy- PID controller are obtained by solving a constrained nonlinear optimization problem, in which the cost function to be minimised is defined by a metric expressed in terms of closed loop system's performance, namely:

subject to:

$$
\min _{K} J(e, u, K) \equiv \min _{K} \sum_{K=1}^{N_{P}} L_{K}(e(k), \Delta u(k), K)
$$




$$
\begin{array}{r}
y(k)-g(k)=0 \\
\phi(y(k), u(k), K) \\
=0 \\
\Psi(y(k), u(k), K) \\
\leq 0
\end{array}
$$

where $J \in \mathbb{R}^{+}$is the performance index, $N_{p} \in \mathbb{R}^{+}$is the prediction horizon, $y \in \mathbb{R}^{p}$ is the output vector, $u \in \mathbb{R}^{m}$ is the control action vector, $K \in \mathbb{R}^{q}$ the vector of scaling factors, $g(\cdot)$ is the nonlinear system dynamics, $\phi(\cdot)$ and $\Psi(\cdot)$ are nonlinear functions associated with the equality and inequality constraints.

Given an initial vector population of scaling factors, the iterative constrained optimization algorithm iteratively calculates a new and improved set of scaling factors, until a minimum of the chosen criterion is reached, within a prespecified time limit or a maximum number of iterations.

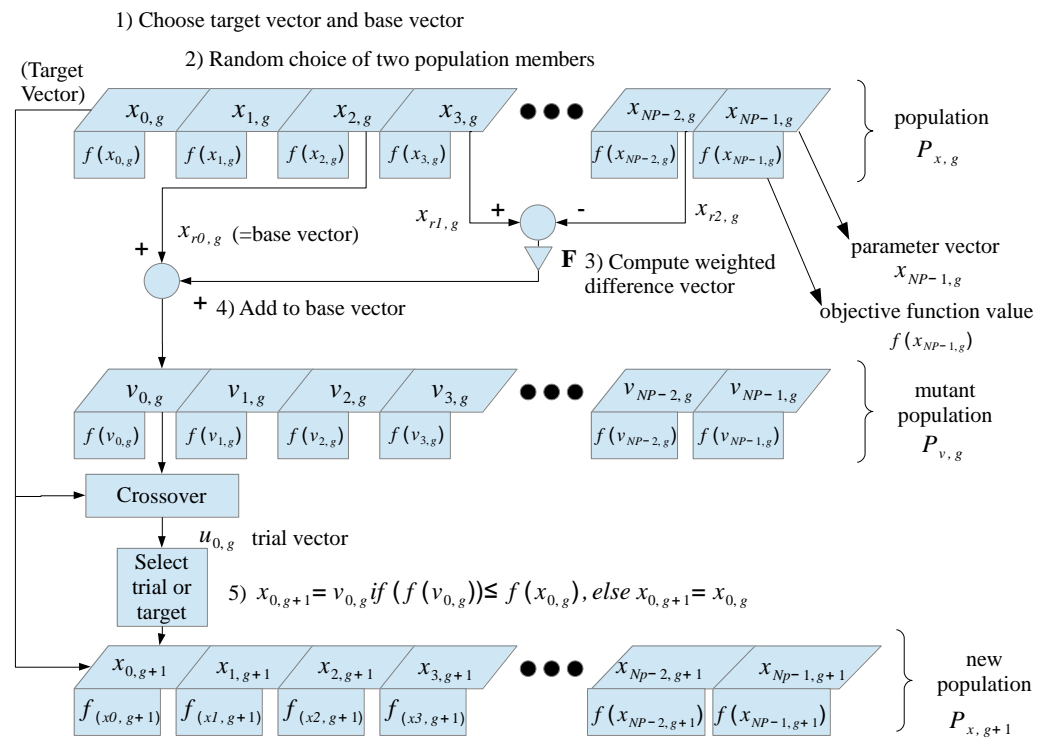

Fig.2: Basic Configuration of Differential Evolution.

Membership Functions Optimization. The approach followed for the membership functions tuning is somehow similar to the one described in Sec. 2-2.2.1, but with two main differences. The first refers to the initialisation of the vector population, which includes the initial values for the width of membership functions, while the second one is related to the fact that at the end of each iteration, both the fuzzy PI and PD components have to be updated. Moreover, constraints on membership functions are also included in underlying optimisation problem. According to Kosko [5], the degree of overlapping between membership functions should lie within the interval [0.25, 0.50 ], while the overlapping rate between membership functions should be exactly 1 , which implies their symmetry. Choosing these values for the degree of overlapping and overlapping rate will contribute to some extent to reduce both the overshoot and the settling time of the closed loop step response.

\section{Cases Studies}

Fuzzy-PID Controller Design. The fuzzy-PID controller comprises two inputs, namely, the control error $\tilde{e}$ and the change in error $\Delta \tilde{e}$, and one output $u^{P I D}$ (see Fig. 1), which is is given by,

$$
u^{P I D}(k)=K_{u} \cdot \Delta \tilde{u}^{P D}(k)+K_{\Delta u} \sum_{q=0}^{k} \Delta \tilde{u}(q)
$$


The normalized universe of discourse for $\tilde{e}$ and $\Delta \tilde{e}$ are chosen as $[-1.5,+1.5]$ and partitioned into seven fuzzy sets (Fig. 3). In the case of the corresponding outputs from the fuzzy PI and PD modules, it was decided that the underlying universe of discourse should also be defined in the range $[-1.5,+1.5]$, while assuming a partition similar to those defined for $\tilde{e}, \Delta \tilde{e}$ and $\tilde{u}$. The membership functions associated with the output $\Delta \tilde{u}$, are represented in (Fig. 4).

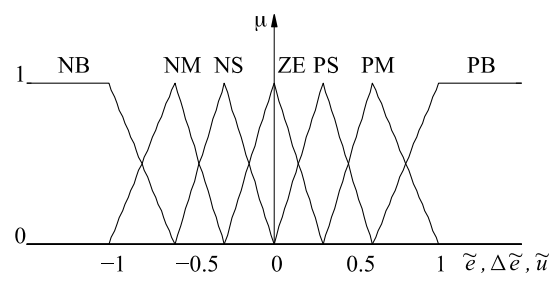

Fig.3: Membership Functions for $\tilde{e}, \Delta \tilde{e}$ and $\tilde{u}$.

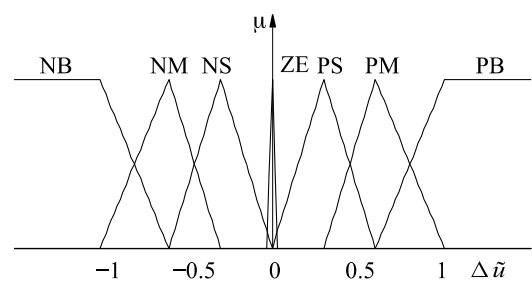

Fig.4: Membership Functions for $\Delta \tilde{u}$.

Experiments. The AMIRA ${ }^{\circledR}$ DTS 200 benchmark three-tank system consists of three plexiglas cylindrical tanks, with identical cross-section, which are supplied with distilled water. The liquid levels $h_{1}, h_{2}$ and $h_{3}$ are measured by piezoresistive transducers in the range of $[-10,+10] \mathrm{V}$. The middle tank $\left(T_{3}\right)$ is connected to the other two tanks through a circular cross-section pipes provided with manually adjustable ball valves, being the main outlet of the system located at tank $T_{2}$.

The cost function was chosen as a quadratic performance index, including penalized control errors and control actions increments. The constrained optimization problem can be written as follows:

$$
\min _{(K, L)}\left\{\begin{array}{c}
\sum_{k=0}^{N_{p}} 20\left[h_{1}(k)-r_{1}(k)\right]^{2}+20\left[h_{2}(k)-r_{2}(k)\right]^{2} \\
+0.1\left[u_{1}(k)-u_{1}(k-1)\right]^{2}+0.1\left[u_{2}(k)-u_{2}(k-1)\right]^{2}
\end{array}\right\}
$$

With respect to the scaling factors vector, $K$, and membership functions width, $L$, they are represented in equation (10) by,

$$
\begin{aligned}
& K=\left[K_{e 1}, K_{\Delta e 1}, K_{\Delta u 1}, K_{u 1}, K_{e 2}, K_{\Delta e 2}, K_{\Delta u 2}, K_{u 2}\right] \\
& \quad L=\left[L_{N B}, L_{N M}, L_{N S}, L_{Z E}, L_{P S}, L_{P M}, L_{P B}\right]
\end{aligned}
$$

They should comply with the following constraints:

$$
\begin{gathered}
0 \leq y(k) \leq 1, k=1, \ldots, N_{p} ; 0 \leq u(k) \leq 5, k \\
\quad=0, \ldots, N_{p}-1 \\
K_{e 1}, K_{e 2} \geq 0 ; K_{\Delta e 1}, K_{\Delta e 2} \geq 0 ; K_{\Delta u 1}, K_{\Delta u 2} \geq 0 ; K_{u 1}, K_{u 2} \\
\quad \geq 0 \\
0.25 \leq \mu_{(N B=N M)} \leq 0.5 ; 0.25 \leq \mu_{(N M=N S)} \leq 0.5 ; 0.25 \\
\quad \leq \mu_{(N S=Z E)} \leq 0.5 \\
0.25 \leq \mu_{(Z E=P S)} \leq 0.5 ; 0.25 \leq \mu_{(P S=P M)} \leq 0.5 ; 0.25 \\
\leq \mu_{(P M=P B)} \leq 0.5
\end{gathered}
$$

The three-tank system is described by a Nonlinear AutoRegressive with eXogenous input (NARX) multilayer neural network consisting of three layers, namely an input layer, a hidden layer and an output layer. Equation (14) represents generically the neural network predictor used in this work.

$$
\begin{gathered}
y_{n e t}(k)=A \cdot \tanh \left[B \cdot U_{n e t}(k)\right] \\
+c
\end{gathered}
$$

In what the membership functions tuning is concerned, in the first approach, where merely the scaling factors are optimised, they are manually selected as shown in Fig. 3 and Fig. 4, while for the 
second approach, both scaling factors and membership functions, are adjusted by means of a DE algorithm so as to minimise the selected performance index. In order to keep the robustness of this approach, in terms of computational time, the membership functions of both the fuzzy PI and PD components are compelled to be the same, except the membership function $\mathrm{ZE}$ of $\Delta \tilde{u}^{P I}$, which is fixed with a very narrow width. In Fig. 5 and Fig. 6 are presented the outcomes of the optimisation procedure.
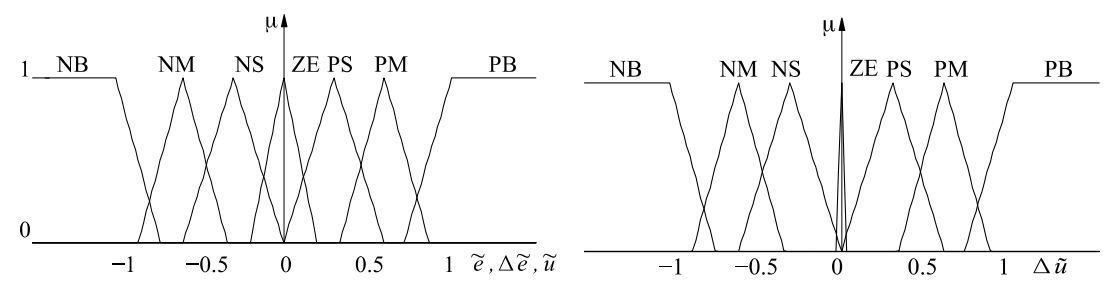

Fig.5: Membership Functions for $\tilde{e}, \Delta \tilde{e}$ and $\tilde{u}$.

Fig.6: Membership Functions

for $\Delta \tilde{u}$.

The results concerning the two methodologies are presented in Fig. 7 and Fig. 8. From visual inspection, one can infer that the underlying closed loop responses are not that much different in terms of outputs. However, focussing the analysis in terms of control action it is clear the outperformance for the optimisation of both scaling factors and membership functions.

The tuning parameters obtained for both approaches are shown in Tables 1 and 2. The widths shown in Table 2 are the same as for both the fuzzy PD and the PI, with the exception of the membership function $\mathrm{ZE}$ of $\Delta \tilde{u}^{P I}$, which is set as 0.06 , and it is not adjusted in the optimisation process.

Table 1: Optimal scaling factors.

\begin{tabular}{|l|c|c|c|c|c|c|c|c|}
\cline { 2 - 9 } \multicolumn{1}{c|}{} & \multicolumn{4}{c|}{ Tank 1 } & \multicolumn{4}{c|}{ Tank 2 } \\
\hline $\begin{array}{l}\text { Appro } \\
\text { ach }\end{array}$ & $K_{e}$ & $K_{\Delta e}$ & $K_{\Delta u}$ & $K_{u}$ & $K_{e}$ & $K_{\Delta e}$ & $K_{\Delta u}$ & $K_{u}$ \\
\hline $\mathbf{K}$ & $\begin{array}{c}0.17 \\
9\end{array}$ & $\begin{array}{c}1.51 \\
5\end{array}$ & $\begin{array}{c}2.04 \\
8\end{array}$ & 8.0 & $\begin{array}{c}0.83 \\
2\end{array}$ & $\begin{array}{c}6.01 \\
4\end{array}$ & $\begin{array}{c}0.59 \\
1\end{array}$ & 8.0 \\
\hline K and & 0.38 & 2.70 & 0.73 & 6.41 & 0.73 & 4.97 & 0.10 & \\
$\mathbf{L}$ & 7 & 9 & 1 & 9 & 7 & 0 & 2 & 7.355 \\
\hline
\end{tabular}

Table 2: Membership functions width.

\begin{tabular}{|l|l|l|c|c|c|c|l|}
\hline Approach & $\boldsymbol{N B}$ & $\boldsymbol{N M}$ & $\boldsymbol{N S}$ & $\boldsymbol{Z E}$ & $\boldsymbol{P S}$ & $\boldsymbol{P M}$ & $\boldsymbol{P B}$ \\
\hline K & 0.80 & 0.60 & 0.60 & 0.60 & 0.60 & 0.60 & 0.80 \\
\hline K and L & 0.53 & $\begin{array}{l}0.53 \\
5\end{array}$ & 0.60 & 0.40 & 0.60 & $\begin{array}{l}0.53 \\
5\end{array}$ & $\begin{array}{l}0.56 \\
7\end{array}$ \\
\hline
\end{tabular}

In order to allow a quantitative assessment of these two approaches in competition, two metrics were used, namely the root mean squared of error (RMSE) and the root mean squared of control action increment (RMSI). The corresponding outcomes are shown in Table 3.

As can be observed from Table 3, the underlying RMSE and RSMI favour the approach in which both scaling factors and membership functions are optimised. This is in line with the analysis based on Fig. 7 and Fig. 8.

Table 3: Performance metrics.

\begin{tabular}{|l|l|l|}
\hline Approach & $\boldsymbol{R M S E}$ & $\boldsymbol{R M S I}$ \\
\hline Scaling Factors Tuning & 0.0572 & 0.0112 \\
\hline Scaling Factors and Membership Functions Tuning & 0.0556 & 0.0104 \\
\hline
\end{tabular}

\section{Conclusions}


This paper addressed the problem of tuning fuzzy PID controllers using a global constrained nonlinear optimisation approach based on a Differential Evolution technique. Two methodologies have been compared: one where only the scaling factors are optimised, while in the other scheme both the scaling factors and membership functions width are optimised. The performance index considered in this study was defined in terms of closed loop performance, and the plant dynamics approximated by a three-layered neural network. Results obtained from two experiments carried out on a benchmark three-tank system show the out-performance of the methodology relying on the optimisation of both scaling factors and membership functions.
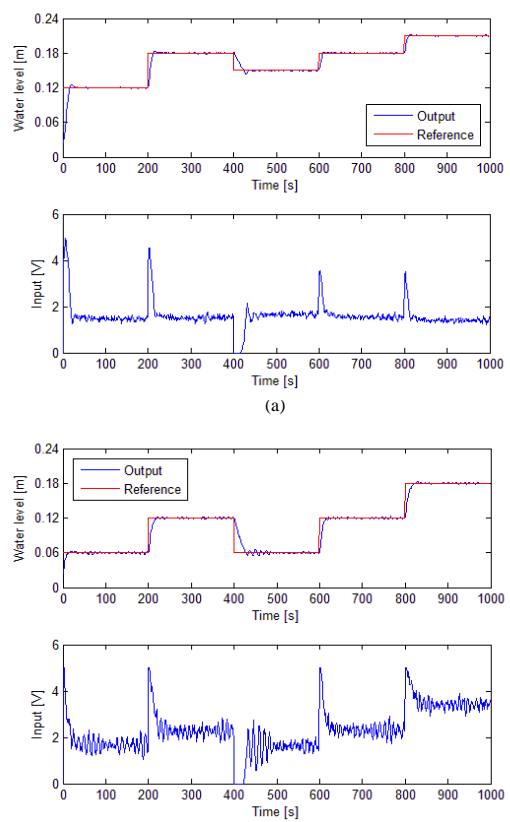

(b)

Fig.7: Fuzzy-PID controller with scaling factors optimization.

a) Tank 1. b) Tank 2 .
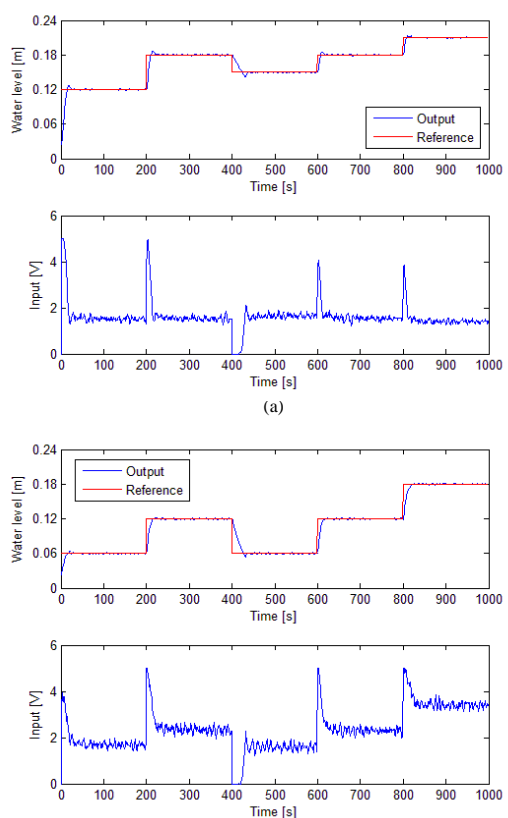

(b)

Fig.8: Fuzzy-PID controller with scaling factors and membership functions optimization. a) Tank 1. b) Tank 2.

\section{References}

[1] G. Feng, "A survey on analysis and design of model-based fuzzy control systems," Fuzzy Systems, IEEE Transactions on, vol. 14, pp. 676-697, Oct. 2006.

[2] P. Gil, C. Lucena, A. Cardoso, and L. Palma, "Gains tuning of fuzzy pid controllers for mimo systems: A performance-driven approach,” Fuzzy Systems, IEEE Transactions on, vol. pp, p. 1, Jun 2014.

[3] R. Storn and K. Price, "Differential evolution-a simple and efficient heuristic for global optimization over continuous spaces," Journal of Global Optimization, vol. 11, no. 4, pp. 341359, 1997.

[4] S. Das and P. Suganthan, "Differential evolution: A survey of the state-of-the-art," Evolutionary Computation, IEEE Transactions on, vol. 15, pp. 4-31, Feb 2011.

[5] B. Kosko, "Fuzzy systems as universal approximators," Computers, IEEE Transactions on, vol. 43, pp. 1329-1333, Nov. 1994. 